\title{
Aplicaciones metodológicas de la educación \\ comparada en investigaciones institucionales
}

\author{
Ducoing Watty, Patricia \\ Instituto de Investigaciones Sobre la Universidad y la Educación, \\ Universidad Nacional Autónoma de México, \\ Ciudad de México, México \\ pducoingw@yahoo.com.mx \\ Rojas Moreno, lleana \\ Facultad de Filosofía y Letras, \\ Universidad Nacional Autónoma de México, \\ Ciudad de México, México \\ ileana_rojas_moreno@hotmail.com \\ Ducoing Watty, Adriana \\ Facultad de Medicina Veterinaria y Zootecnica, \\ Universidad Nacional Autónoma de México, \\ Ciudad de México, México \\ adriana.ducoing@gmail.com
}

\section{Resumen}

La presente comunicación, derivada de los avances de investigación de los proyectos institucionales PAPIIT IN-402712, IN-403813 e IN-401218, ofrece algunas consideraciones teórico-metodológicas sobre la Educación Comparada aplicables a dos objetos de estudio en particular: la educación secundaria y la incorporación de las Tecnologías de la Información y la Comunicación (TIC) en la educación superior. En primer lugar, argumentamos la importancia de utilizar la estrategia comparativa en la investigación educativa desde un encuadre que combine la perspectiva sociohistórica con precisiones de orden teórico y metodológico, tomando como guía las categorizaciones de Estado-nación, Sistema-mundo, gobernanza, política educativa, sistemas educativos, internacionalización y virtualización de la educación, en cuyo desarrollo incorporamos aportes de autores expertos en este campo de conocimiento, en el cual la aproximación comparativa define uno de los ejes de búsqueda y obtención sistemática de evidencias para la construcción de objetos de investigación. En segundo lugar y a manera de resaltar el potencial de los estudios comparativos, incluimos algunas consideraciones relevantes derivadas de las investigaciones de referencia, destacando señalamientos de orden metodológico y algunos avances obtenidos de dicha aplicación. En el caso de la educación secundaria y mediante la selección justificada de países de Europa y América Latina, observamos que este ciclo educativo se configura como un objeto único y singular en el panorama de la diversidad, y al mismo tiempo como un espacio formativo común, institucionalizado internacionalmente en tanto un nivel de estudios de la educación básica. En cuanto al caso de la incorporación de las TIC en la práctica educativa, utilizamos la categoría de educación superior en modalidades no presenciales y la metodología comparativa destacando la articulación entre el panorama internacional y la oferta educativa en el contexto mexicano, a fin de elaborar un diagnóstico que posibilite visualizar tendencias y desafíos en la composición de esta vertiente de atención educativa.

\section{Abstract}

The present communication, derived from the research advances of the institutional projects PAPIIT IN402712, IN-403813 and IN-401218, offers several theoretical-methodological considerations on Comparative Education applicable to two study objects in particular: secondary education and the incorporation of Information and Communication Technologies (ICT) in higher education. Firstly, we argue the importance of using the comparative strategy in educational research from a framework that would combine the sociohistorical perspective with precisions of theoretical and methodological order, taking the categorizations of nation-state, public policies, educational systems, internationalization and virtualization of education, among others, as a guide. We also incorporate the contributions of expert authors in this field of knowledge, in which the comparative approach defines one of the axes of search and systematic collection of evidence for the construction of research objects. Secondly, and in order to highlight the potential of comparative studies, we include some of the advances obtained in the reference investigations, highlighting the most representative results of this application. In the case of secondary education and through the justified se- 
lection of some countries in Europe and Latin America, we observe that this educational cycle is configured as a unique and singular object in the panorama of diversity, and at the same time as a common formative space, institutionalized internationally as a level of studies of basic education. Regarding the incorporation of ICT in educational practice, we use the category of higher education in remote modalities and a comparative methodology, highlighting the articulation between the international panorama and the educational offer in the Mexican context, in order to elaborate a diagnosis that makes it possible to visualize trends and challenges in the composition of this aspect of educational service.

Palabras clave: educación comparada; metodología comparativa en educación; educación secundaria; educación superior; Tecnologías de la Información y la Comunicación (TIC).

Keywords: comparative education; comparative method in education; secondary education; higher education; Information and Communication Technologies (ICT).

\section{INTRODUCCIÓN}

En opinión de expertos (Schriewer, 2011; Schriewer y Pedró, 1993; Valle, et al., 2016, entre otros), la Educación Comparada ha avanzado notablemente en lo que se refiere al desarrollo de enfoques teóricos y propuestas metodológicas. De hecho, durante las últimas décadas y de manera gradual las investigaciones de corte comparativo en educación han adquirido una mayor presencia, tomando en cuenta el marco configurado por los procesos de internacionalización y globalización económica y su derivación en el planteamiento de nuevos problemas soportados en encuadres teóricos cada vez más elaborados y con volúmenes de datos que aumentan exponencialmente de un momento a otro. Para efectos de la presente comunicación, nuestro propósito va más allá de proponer tan solo una visión instrumental de la metodología de corte comparativo, pretendiendo una reapropiación por decreto de la Educación Comparada por parte de las instituciones académicas, por lo cual diferenciamos dos tareas a cumplir.

En primer lugar, argumentamos la importancia de utilizar la estrategia comparativa en la investigación educativa desde un encuadre que combine la perspectiva sociohistórica con precisiones de orden teórico y metodológico, tomando como guía las categorizaciones de Estado-nación, Sistema-mundo, gobernanza, políticas públicas, sistemas educativos, internacionalización y virtualización de la educación. Es así que, a partir de una demarcación basada en desarrollos teóricos de autores seleccionados, consideramos la pertinencia de utilizar la aproximación comparativa definiendo algunos ejes de búsqueda y obtención sistemática de evidencias para la construcción de objetos de investigación. En segundo lugar incluimos algunas consideraciones relevantes derivadas de tres investigaciones institucionales, destacando señalamientos de orden metodológico y algunos de los avances obtenidos de dicha aplicación que dan cuenta del potencial de los estudios de esta índole.

\section{NOTAS PRELIMINARES SOBRE CATEGORIZACIONES}

En este apartado reflexionamos sobre las categorizaciones anteriormente mencionadas mismas que, a manera tanto de intuiciones teóricas como de herramientas analíticas, nos permitan establecer los soportes y límites para construir objetos de investigación.

\subsection{Notas sobre Estado-nación y Sistema-mundo}

El interjuego en la aplicación de estas dos categorizaciones da cuenta de un cambio de óptica en los estudios comparados, sobre todo si partimos de situar la categoría de Estado-nación como uno de los puntos de inicio e impulso para la Educación Comparada, en tanto que el manejo de la categoría de Sistema-mundo lo ubicamos como recurso en la producción de conocimientos con miradas multirreferenciales. Conviene destacar que cada una de ellas ocupa unidades de análisis diferenciadas; por ejemplo, en el caso de Estado-nación ubicamos un esquema representativo de un territorio y/o una entidad social y políticamente responsable de las formas de organización, participación y convivencia de las personas en una pretendida homogeneidad cultural, económica y social. Por su parte, en el 
caso de Sistema-mundo como unidad de análisis para el estudio de lo social, nos referimos a una figura estructurante relacionada con la incorporación por parte de las ciencias sociales de los fenómenos de internacionalización y globalización. De acuerdo con Wallerstein (2013), este posicionamiento representa un cambio más apropiado para aproximarse a las visiones globales y de interdependencia. Citamos: "Los sistemas-mundo de análisis significaron antes que nada la sustitución de una unidad de análisis llamada "sistema-mundo" en vez de la unidad estándar de análisis, que había sido el estado nacional. [...] En lugar de los estados nacionales como objetos de estudio, los sustituyeron por «sistemas históricos» que [...] habían existido hasta ese momento en sólo tres variantes; mini sistemas, y «sistema-mundo» de dos tipos (economías-mundo e imperios-mundo) (Wallerstein, 2013: 32).

Derivado de estas diferenciaciones resalta entonces el debate sobre el alcance de los análisis de la globalización frente a la cobertura de los análisis nacionales. Según Schriewer (2011), no se trata de una oposición excluyente, sino justo de un interjuego de categorizaciones dejando entrever que los procesos de internacionalización requieren necesariamente la existencia de los Estados nacionales, vistos como unidades sociales, culturales y políticas. Cabe recordar que en el corpus discursivo de la Educación Comparada no fue sino hasta finales del siglo XX y principios del nuevo milenio cuando fue posible apreciar cómo, de una visión de mundo concebido como una multitud de sociedades regionales o nacionales separadas, se ha cambiado a la visión de configuraciones históricamente caracterizadas e interdependientes.

\subsection{Notas sobre gobernanza}

Para el manejo de la categorización de gobernanza partimos de las siguientes consideraciones. De entrada, podemos apreciar que en la actualidad la discusión acerca de los gobiernos democráticos se enfoca en la capacidad para dirigir y conducir eficazmente una sociedad, es decir, en el impacto y beneficio social de sus decisiones y resultados para encarar los problemas y procesar las demandas sociales. De acuerdo con Aguilar (2010), bajo esta lógica el énfasis no se concede al tema del gobernante, sino al de la manera de gobernar, en tanto que no está cuestionada la legitimidad de su cargo sino la pertinencia de sus decisiones y acciones, su competencia, en suma, su capacidad directiva. Observamos entonces que se trata de un desplazamiento del sujeto gobernante hacia la acción del gobierno o de gobernanza.

Incorporada desde hace ya varias décadas al mundo de la política, de la sociología, de la ética, de la administración, del derecho y de la gestión, esta categorización constituye un principio teórico a la vez que pragmático, utilizado para explicar y hacer frente a los retos que afronta el Estado en su relación con los actores, convirtiéndose así a partir de los años noventa «...en el símbolo de una nueva modernidad en los modos de acción pública» (Theys, 2003: s/p). La gobernanza, por tanto, alude a los cambios en las formas de gobierno -asociados con la transparencia y la ética, igual que con la eficiencia de la acción pública- que varían de acuerdo con los actores y los contextos institucionales, cambios que, suponemos, tienden a generar decisiones más democráticas y funcionales. Si bien no existe una acepción única para esta categorización, sí se puede establecer su vinculación con otros conceptos cercanos, tales como gobernabilidad, Estado, gestión pública, entre otros, los cuales delimitan el contorno de una misma cuestión: la de la conducción de la sociedad.

Derivado de lo anterior, afirmamos que la utilización de dicha categorización -en el abordaje de lo educativo a nivel local, nacional e internacional- permite replantear cuestiones sobre las relaciones comúnmente identificadas entre el gobierno y el sector de educación, incluyendo así la relativa independencia con que se desempeña cada maestro, cada centro escolar, cada supervisor o inspector, cada autoridad territorial, esto último dados la capacidad y los recursos de que disponen todos estos actores para conducir y operar los diferentes servicios educativos.

\subsection{Notas sobre política educativa}

La categorización de política educativa, entretejida con las conceptualizaciones de política y política pública, representa uno de los basamentos-guía para una aproximación comparativa entre los planos nacional, regional e internacional, a modo de herramienta para contextualizar las dinámicas de participación de comunidades e instituciones propiciadoras de espacios de interacción cotidiana para la transformación y puesta en práctica de la política educativa. Recordemos que dada la polisemia 
del concepto de política es necesario considerar algunas precisiones. Una de ellas y de acuerdo con Plano (citado por Aguilar, 2014: 24), alude a una definición mediante la cual usualmente se ubica «... un conjunto o secuencia de decisiones [...] más que una decisión singular acerca de una acción de gobierno particular. Algunos la entienden como decisiones de fines [...]. Otros, en contraste, consideran que la política incluye los medios y los fines. En algunos contextos denota decisiones [...] de largo plazo [...] o directrices generales de acción gubernamental que guían las acciones de corto plazo... «. Otra consideración importante es la de tomar en cuenta la diferenciación entre política (politics) y política(s) (policy/policies), al enfatizar que el concepto política está relacionado en general con un esquema de regulación aplicable a un sistema. En suma, mientras que la política (politics) incluye en un sentido genérico poder, intereses, competencia, conflicto y representación, las políticas (policies), concretamente las políticas públicas (public policies) se enfocan en el debate y la definición de líneas y/o cursos de acción en función de problemas debidamente acotados.

En relación con estas conceptualizaciones ubicamos la categorización de política educativa misma que, de manera recurrente tanto en documentos de investigación como en documentos prescriptivos, revela la estrecha articulación existente entre la sociedad, la economía, la política y desde luego la educación, la cual podemos situarla en términos de un conjunto complejo y heterogéneo de procesos histórico-sociales con funciones específicas (socializadora e ideológica, cultural, académica, distributivo-selectiva, control y regularización social, económica y formadora de la fuerza de trabajo, ocupacional, investigativa). Para abreviar, toda vez que la educación queda a cargo del Estado, la intervención "estatal» habrá de considerarse en términos de política educativa, categorización que definiremos entonces como el conjunto de acciones del Estado con el fin de estructurar y regular el sistema educativo.

\subsection{Notas sobre sistemas educativos}

Investigadores y expertos tanto nacionales como internacionales coinciden en posicionar la Educación Comparada como un ámbito destinado al estudio de los sistemas educativos de los diferentes países del Orbe. Desde los inicios de la Era Moderna, el surgimiento de los sistemas educativos en los Estados nacionales más desarrollados del continente europeo sentó un precedente en lo referente al estudio de los procesos fundacionales de instituciones educativas de diferentes niveles y grados, situación que al paso de los siglos derivó en la estructuración de sistemas educativos, de todos sus niveles, grados y modalidades.

Desde nuestra perspectiva, el manejo de esta categorización como herramienta de análisis nos permite ubicar históricamente la emergencia y consolidación de modelos racionalizados del Estado-nación y de la educación de masas y de élite en una visión secular, al considerar que este conjunto de procesos ha propiciado el establecimiento de instituciones más homogéneas en los distintos países. Desde una perspectiva histórica observamos que los modelos educativos mundiales han influido decisivamente en la reconfiguración de los sistemas educativos nacionales, proclives a una homogeneización relativa y una estandarización en aumento, lo anterior sin perder de vista la participación creciente de las organizaciones internacionales y de las ciencias y profesiones educativas, y de manera cada vez menos directa desde las naciones importantes hacia las periféricas (Schriewer, 2011).

\subsection{Notas sobre internacionalización y virtualización de la educación}

En el encuadre de la llamada globalización económica conviene diferenciar procesos específicos, tal y como precisamos las categorizaciones de este rubro. Por ejemplo, mediante la categorización de internacionalización ubicamos las complejas dinámicas geopolíticas tendentes a la intensificación de los procesos globales de interacción, intercambio, interconexión y armonización trasnacional de modelos y estructuras sociales. Otra vez de acuerdo con Schriewer (2011), nos posicionamos frente a un fenómeno de expansión permanente y de impacto en todos los ámbitos de la interacción social, influyendo inevitablemente en la configuración de los sistemas educativos. Ejemplos concretos de este conjunto de fuerzas los encontramos en programas de movilidad académica, en la integración de innovaciones tecnológicas, o bien en modelos educativos no presenciales estandarizados, por mencionar algunos de ellos. 
En cuanto a la categorización de virtualización de la educación, utilizamos esta precisión para abarcar una dinámica de incorporación de las Tecnologías de la Información y la Comunicación (TIC) para incrementar la oferta de los servicios educativos en los diferentes grados y niveles de los sistemas educativos de todo el mundo. De acuerdo con Rama (2012), este proceso cobró auge en la década de los años noventa aparejándose con la digitalización global de la economía y la sociedad, al entreverarse con factores como el requerimiento de la calidad en la enseñanza y el aprendizaje, la disminución de costos de los servicios educativos, la segmentación de niveles y modalidades y la ampliación exponencial de la cobertura de escolarización, entre otros. El elemento clave para esta forma de expansión educativa ha sido y es el uso por excelencia de la Internet, en tanto que la diversidad nominativa -léase educación abierta, educación a distancia, educación en línea, educación no presencial- incluye una amplia gama de recursos tales como redes, plataformas, softwares, repositorios, cursos masivos, entre otros. En el entretejido de esta contextualización cabe resaltar también la configuración de nuevos marcos normativos, académico-institucionales y, por supuesto, pedagógicos.

\section{EDUCACIÓN COMPARADA E INVESTIGACIÓN INSTITUCIONAL}

El encuadre institucional brindado por la Universidad Nacional Autónoma de México ha sido el espacio de trabajo para el desarrollo de tres proyectos de investigación vinculados con la Educación Comparada, en los que a partir de actividades como conformación de equipos de trabajo, realización de seminarios de discusión teórico-metodológica, participación foros y eventos nacionales e internacionales para la divulgación de resultados, entre otras, hemos refrendado nuestro compromiso académico con la investigación ${ }^{1}$.

\subsection{Derivaciones metodológicas}

La primera fase en la estructura y organización de cada una de las investigaciones indicadas consistió en definir esquemas panorámicos con los diferentes niveles de comparatividad (p. ej., internacional, regional, nacional, local, institucional) desde los cuales, y mediante la utilización de categorías analíticas vinculantes (p. ej., currículum de educación secundaria, incorporación de TIC en la práctica docente, modalidades no presenciales), situamos los planos de la investigación (p. ej., inter e intraniveles) así como los entrelazamientos posibles. La segunda fase implicó la definición de estrategias de acopio, sistematización y presentación descriptiva de información documental (gabinete), empírica (estadística) y/o de campo (observaciones, entrevistas). La tercera fase requirió la selección de las categorías de análisis pertinentes para demarcar redes de interrelación intra e interniveles, tomando como guía los desarrollos teóricos señalados en el primer apartado de este documento. En la cuarte fase desarrollamos ejercicios de contraste entre unidades de comparatividad (estudios de caso de países, sistemas educativos, formas de gobernanza en comunidades y/o instituciones participantes, caracterización de modalidades no presenciales, prácticas docentes apoyadas en el uso de TIC). En la quinta fase, aún en desarrollo, nos hemos abocado a establecer y afinar algunas vinculaciones hipotéticas entre los aspectos destacados de una determinada categoría (p. ej., en el caso de una política educativa específica, ubicar sus alcances, impacto y efectos), resaltando la vinculación entre los diferentes niveles (internacional, regional, nacional, local, institucional) y los contextos socioculturales correspondientes (región, país, cultura, institución). En la sexta y última fase, con la cual concluiremos los proyectos de referencia, habremos de mostrar el entretejido de este conjunto de articulaciones empíricas y las argumentaciones hipotéticas de cada uno de los objetos de investigación construidos. Con este cierre pondremos a prueba la capacidad de generalización teórica y de explicación y/o comprensión del conjunto de estrategias elegidas.

\footnotetext{
${ }^{1}$ Los Proyectos de referencia forman parte del Programa de Apoyo a Proyectos Institucionales de Investigación y Tecnología (PAPIIT), y se registraron con las claves siguientes: IN402712, IN403813 e IN401208.
} 


\subsection{Proyectos y avances de investigación}

Presentamos a continuación algunos de los rasgos más relevantes de los proyectos de investigación institucionales mencionados.

a. Proyecto PAPIIT IN402712 «La Educación Secundaria: retos y perspectivas al inicio del siglo. Estudio comparado».- Iniciado en 2012, este proyecto tiene como objetivo principal de analizar la situación de la educación secundaria en diversos países de Latinoamérica y Europa. Las preguntas de investigación fueron las siguientes: a) ¿cuáles son las tendencias internacionales en torno a la educación secundaria?; b) de acuerdo con el vínculo entre política educativa y currículum, ¿qué rasgos se comparten y cuáles particularizan en cada caso este nivel educativo?; $y, c)$ ¿de qué manera los contextos nacionales han influido en la configuración del currículum de la educación secundaria en el panorama internacional? Los desarrollos obtenidos han posibilitado la construcción de una visión panorámica y comparativa sustentada en la preparación de estudios de caso. El eje transversal quedó definido a partir de la categoría de «currículum de educación secundaria» y su caracterización en países como Argentina, Austria, Bélgica, Brasil, Bolivia, Chile, Colombia, Costa Rica, España, Francia, Guatemala, Inglaterra, México, Perú, Venezuela y Uruguay.

b. Proyecto PAPIIT IN402712 «Panorama comparativo sobre el manejo de las TIC en la práctica docente del SUAyED-FFyL-UNAM».- Desde 2013, el proyecto tiene como objetivo realizar una caracterización panorámica comparativa sobre la práctica docente de los profesores de la División SUAyED/FFyL, contextualizada en el marco del impacto de la incorporación de las TIC en la implantación de modalidades no presenciales en educación superior. Las preguntas de investigación fueron las siguientes: a) ¿cómo se caracteriza la educación superior en modalidades no presenciales en el panorama internacional?; b) de acuerdo con las particularidades sociohistóricas y económicas de nuestro país, ¿cuál ha sido la influencia internacional en la configuración de este subsistema en México?; y, c) a partir de esta influencia ¿cómo se caracteriza la práctica docente en el SUAyED tomando en cuenta el uso de las TIC? De los avances obtenidos destacamos el análisis comparativo centrado en abordar los rasgos predominantes de la tendencia actual de virtualización de la educación superior, tal y como viene sucediendo en México desde hace poco más de cuatro décadas, así como de los estilos de docencia apoyados en el uso de las TIC en la entidad en cuestión.

c. Proyecto PAPIIT IN401208 «La educación secundaria europea y latinoamericana. Un estudio comparado».- Comenzado en 2017, este proyecto tiene como objetivo analizar, desde una perspectiva comparada, la educación secundaria europea y latinoamericana de cara a la problemática actual, con especial énfasis en aspectos como agendas internacionales de políticas educativas, proyectos curriculares y tendencias de formación docente (inicial y continua). Las preguntas de investigación que guían el proyecto son las siguientes: a) ¿cómo se caracteriza la educación secundaria en el panorama internacional?; b) de acuerdo con las particularidades sociohistóricas y económicas de cada país, ¿cuáles son las semejanzas y diferencias de este nivel educativo?; y, c) ¿cuál ha sido la influencia de los contextos nacionales en la configuración del currículum del nivel educativo en cuestión? Por tratarse de un trabajo aún incipiente, contamos apenas con datos procesados que muestran rasgos como los siguientes: a) la tendencia de la educación secundaria a universalizarse, particularmente en Latinoamérica, al haberse decretado su obligatoriedad; $y, b)$ su naturaleza ambigua e indefinida al interior los diversos sistemas educativos, como por ejemplo, continuidad de la primaria, preparación para el bachillerato, opción técnica (para la incorporación al mercado laboral).

\section{DISCUSIÓN Y CONCLUSIONES}

A partir de las experiencias de investigación reafirmamos la importancia de abordar la gama de posibilidades de la metodología de investigación comparada en educación como uno de los recursos heurísticos para descubrir nuevos retos y estrategias de búsqueda. Mediante las puntualizaciones desarrolladas visualizamos diferentes horizontes de búsqueda. 
Concluiremos diciendo que en los tres proyectos de referencia nos hemos propuesto desarrollar ejercicios de comprensión desde el interior de los sistemas y modelos educativos --signados por la historicidad y el contexto específico de los respectivos objetos de investigación - resaltando semejanzas y diferencias desde marcos interpretativos que posibiliten aproximaciones al sentido que se le puede asignar a los hechos y fenómenos que caracterizan las diferentes unidades de análisis, esto último con base en las categorizaciones establecidas.

Por último, insistimos que las tensiones entre universalismo y particularismo, homogeneidad y heterogeneidad, singularidad y pluralidad, diferencia y similitud -entre otros posicionamientos aparentemente opuestos - se entretejen estas dimensiones constitutivas desde una óptica de la dualidad que se inscribe en la historicidad como exigencia irreductible, al reconocer las dinámicas que caracterizan las articulaciones prevalecientes entre una mirada universalizante y un panorama particular y viceversa, entre un contexto homogéneo y uno diverso.

\section{REFERENCIAS BIBLIOGRÁFICAS}

AGUILAR-VILLANUEVA, L. F. (2010), Gobernanza. El nuevo proceso de gobernar (México, Fundación Friedrich Naumann para la Libertad).

AGUILAR-VILLANUEVA, L. F. (2014). Estudio introductorio, en La hechura de las políticas, pp. 15- 84 (México, Miguel Ángel Porrúa/Colección Antologías de Política Pública, Segunda antología).

DUCOING-WATTY, P. (2012): Proyecto PAPIIT Clave IN 402712 La Educación Secundaria: retos y perspectivas al inicio del siglo. Estudio comparado (México, UNAM/Documento institucional).

DUCOING-WATTY, P. (2017): Proyecto PAPIIT Clave IN 401208 La educación secundaria europea y latinoamericana. Un estudio comparado. Estudio comparado (México, UNAM/Documento institucional).

ROJAS-MORENO, I. (2013): Proyecto PAPIIT Clave IN 403813 Panorama comparativo sobre el manejo de las TIC en la práctica docente del Sistema Universidad Abierta y Educación a Distancia-Facultad de Filosofía y Letras-UNAM (México, UNAM/Documento institucional).

THEYS, J. (2003), «La Gouvernance entre innovation et impuissance», Développement durable et territoires, Dossier 2 , (http://journals.openedition.org/developpementdurable/1523) consultado el 23 de marzo de 2018.

SCHRIEWER, J. (2011): Sistema mundial y redes de interrelación: la internacionalización de la educación y el papel de la investigación comparada, en CARUSO, M., \& TENORTH, H-E. (Comps.), Internacionalización. Políticas educativas y reflexión pedagógica en un medio global, pp. 41-105 (Buenos Aires, Granica).

SCHRIEWER, J. \& F. PEDRÓ (Eds.), Manual de educación comparada (Vol. II) Teorías, investigaciones, perspectivas (Barcelona, Pomares-Corredor).

RAMA, C. (2012). La reforma de la virtualización de la universidad. El nacimiento de la educación digital (México, Universidad de Guadalajara Virtual) (http://virtualeduca.org/documentos/observatorio/libro_la-reforma-de-la-virtualizacion-de-la-universidad-claudio-rama-udg-2012.pdf) consultado el 16 de abril de 2017. 
VALLE, J. M. et al. (2016): Investigación en Educación Comparada: Pistas para investigadores noveles, Revista Latinoamericana de Educación Comparada, 7 - 1, pp. 39-56 (http://www.saece.org.ar/relec/revistas/9/revista_a7n9.pdf\#page=39) consultado el 18 de mayo de 2017.

WALLERSTEIN, I. (2013): Análisis de sistemas-mundo. Una introducción (México: Siglo XXI). 